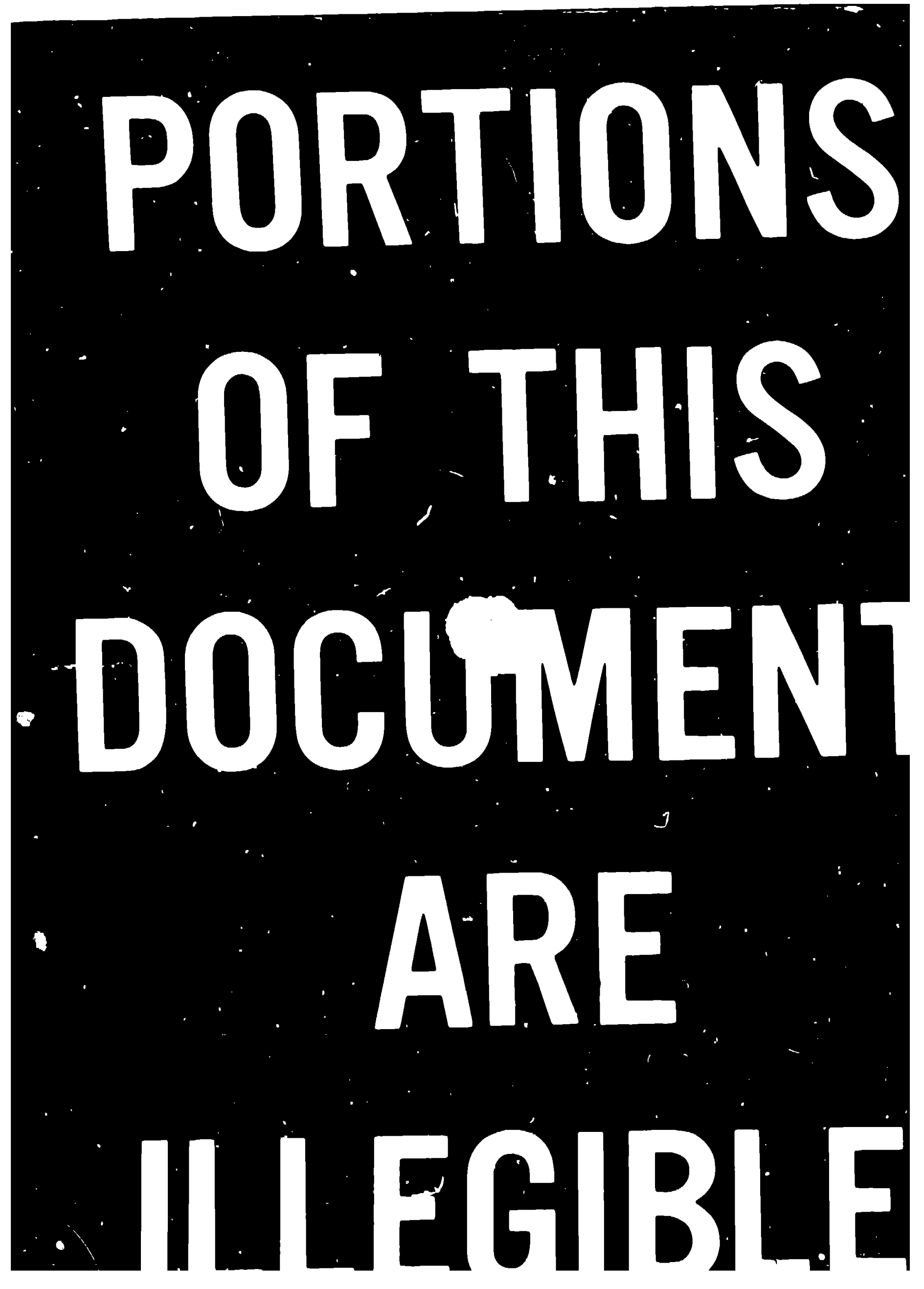


LA-UR -82-820

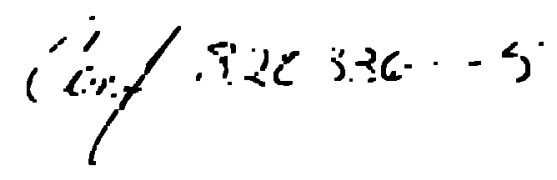

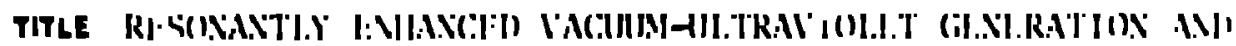

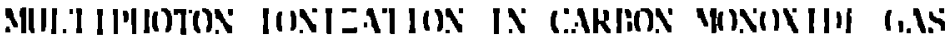

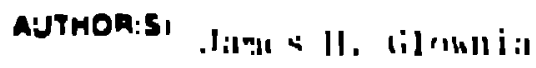

kiluelel f.. Silnder
}

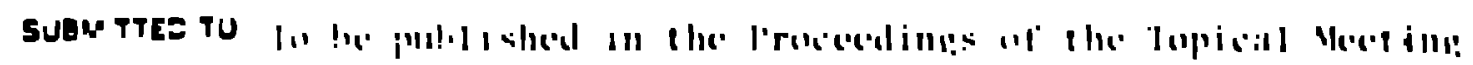

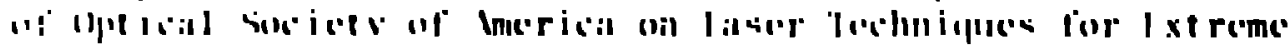

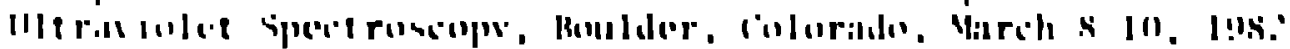




\title{
RESONANTLY ENHANCED VACUUM ULTRAVIOLET GENERATION AND MULTIPHOTON IONIZATION IN LARBON MONOXIDE GAS*
}

\author{
James $H$. Glownia and Robert $K$. Sander \\ University of California, Los Alamos National Laboratory \\ Los Alamos, New Mexice 87545
}

\begin{abstract}
Competition between three-photon resonantly enhanced vacuum ultraviolet third-harmonic generation and six-photon multiphoton ionization using the $A$ state in gaseous carbu monoxide is observed. Excitation spectra of the third-harmonic emission exhibit increasing blue shifts and broadenir.g with increasing pressure due to the phase matching requirements. Estimates for the efficiency and turability show that third-harmonic generation in carbon monoxide molecules is is promising source for coherent vacuum ultraviolet light.
\end{abstract}

\section{INTRODUCT ION}

The attraction of using molecular vapors as a nonlinear medium to Renerate vacuum ultraviolet (lill) radjation has recellly bech revieved hy willace.' Despite the ronvenience of hiving a fixed gats for the nonlinear medium, single frequency pump sources, anll bide tunability due to the width and number of molecular vibronic binds, only one detailed account of third-harmonic generation (TllG) in molecules has been published.2 In that study, tho and threeplotoll resonantly enhanced THG in nitric oxide was reported. We report the ohservation of three-photon resonintly enhanced THG through the $A^{1} \mid l$. $X^{1} 2$ transition (fourth positive system) of cartion monoxide (CO) gas. In addition to detecting Vul radiation we simultannously recoril signals trom A'ח-resonantly enhanced six-photon multiphoton ionizalion (NPI). Kesules of such studies in xenon ${ }^{3.4}$ have shown that THG and MPI can be compelitive processes. We report similar lindings where it is observed that signals due 10 MPJ disappear al wavelengths to the blue of the R-head in the CO A state and, concurrently, intense VII' third-harmonic radiation is delected in the forward direction.

Despite the fact that nonresonant THG in C.o has failed in the past, il was chosen in the present study for a number of reasons. It is a gascous material and processes competing with third-harmonic emission are suppressed. Its high ionizat ion potential (14.01 (1/ $)^{\prime \prime}$ and high dissocialio.l limit (11.11 cV) ${ }^{7}$ make these two processes higher order and tlerefore unlikely in the wilvelength region ul interest. Furthermore, it was Irlt that by using the resollillt enthancement due $\mathrm{en}$ the strong transition dipole of the $A$ stile, ill cfficient lill radiation source could in produced using powerful dye-laser pump, sources. Finally, the extensive Frinck-condon churelope of this transition provides a wide tunillg range.

ir

Work performed under the aluspices of the US IM)ti. 


\section{EXPERIMENTAL}

The experimental apparatus, shown in Fig. l, is similar to that used by Miller et al. ${ }^{3}$ It consists of a four-way cross connected to a vacuum system. The output of a frequency-tripled Nd: YAG laser (Quanta-Ray DCR-1) pumped Colmarin 440 dye laser (Quanta-Ray PDi-1) is focused by either a $75-$ or $100-\mathrm{mm}$ focal length lens to a haist calculated to be about $10 \mathrm{\mu m}$. The laser flux in the focal voluifit wiats on the order of $10^{29-10^{30}}$ photons $\mathrm{cm}^{-1} \mathrm{~s}-1$. Photoelectrons risultinf from the MPl process are monilored with a flat-plate platunum collector. Amplification occurs by an electron avalanche in the lil gas lale to a negalive hias of 200-300 l'on the otlicr platinum plate. lacuull UV ladiation can pass through a lif nindow into a differentiall!: pumped live-hay cross filled with argon cuunting gas at 0.5 Torr. ll

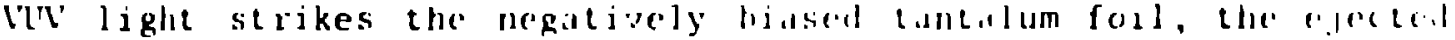
photoelectrons are collected with amolher llat-plate platiludile ele-

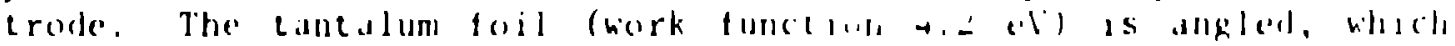

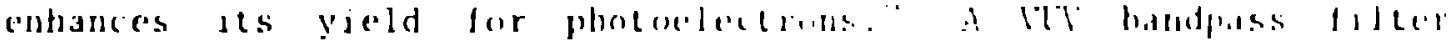

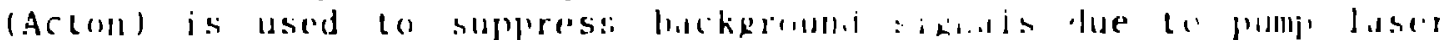

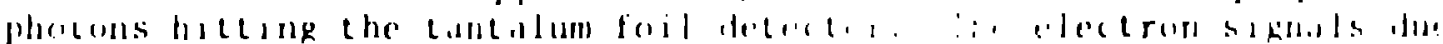

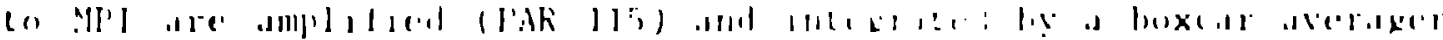

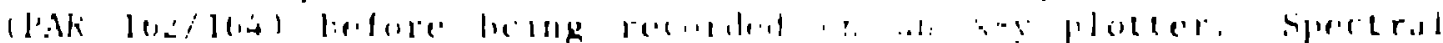

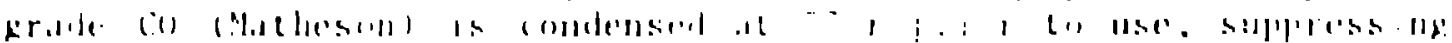

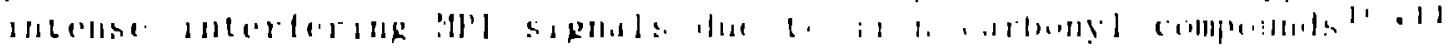

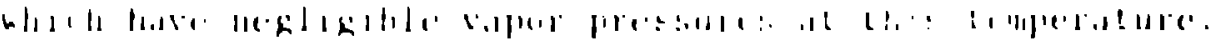

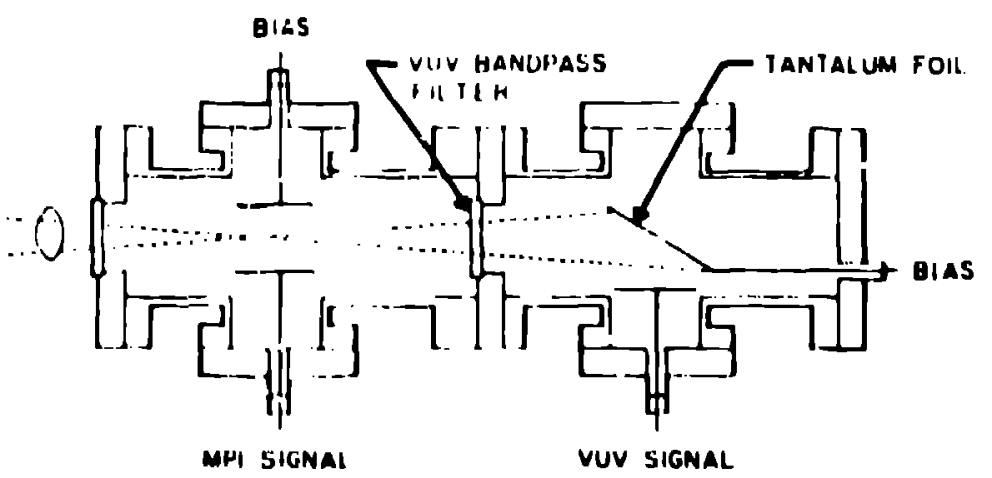

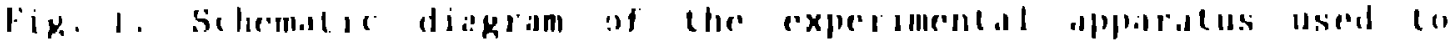

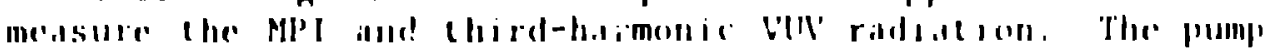
liser rall also be positeuned al q00 with respece to the detectior.

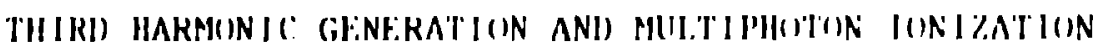

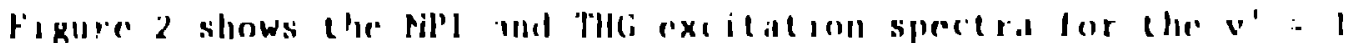

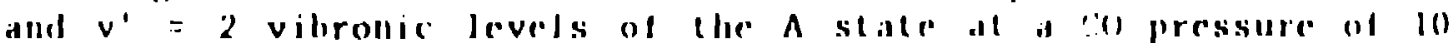
Turr. The innizalion sighals are due lo threepholon All resonint ly chlialled six-photon Mll. The signils al slower laser wilvelength scill 
speeds show the anticipated rotational struclure. ${ }^{k}$ To the h] ue of the R-head, ionizalion signals ran no longer be detected, and only signals due to VUV radiation are observed. The VUV light is oniy detected in the forward direction with respect to the pumj lasci. The excitation spectrum exhibits increasing blue shifts and hintwidths with increasing pressure. This is shown in Fig. 3 . $\therefore$ lso apparent in Fig. 3 are sharp dips in the llit signal due to ahserpticil by e ${ }^{3} \Sigma^{-}\left(v^{\prime}=4\right)$ rovibronic levels in $\mathrm{CO}$," confirming identificuliur the IV light as tunable monochromalis third-harmonic enisstell. 1 . account for sinilar MPI-THG pnenomena in xchull, a two-level merlf.l li.l: been used.' Th:s two-level model involves coherent excitation rit ., group of atoms resulting in a collection of coupled dipules klicl. emit to the ground stale with an enhancred costllator strength. !!ia!-

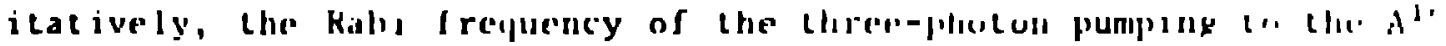

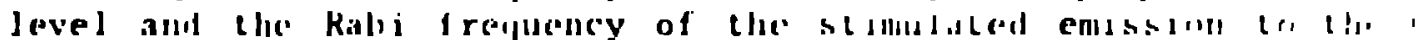
Rrounil stale arie coupleil.

COMPETITION GETWEEN MPI AND THG

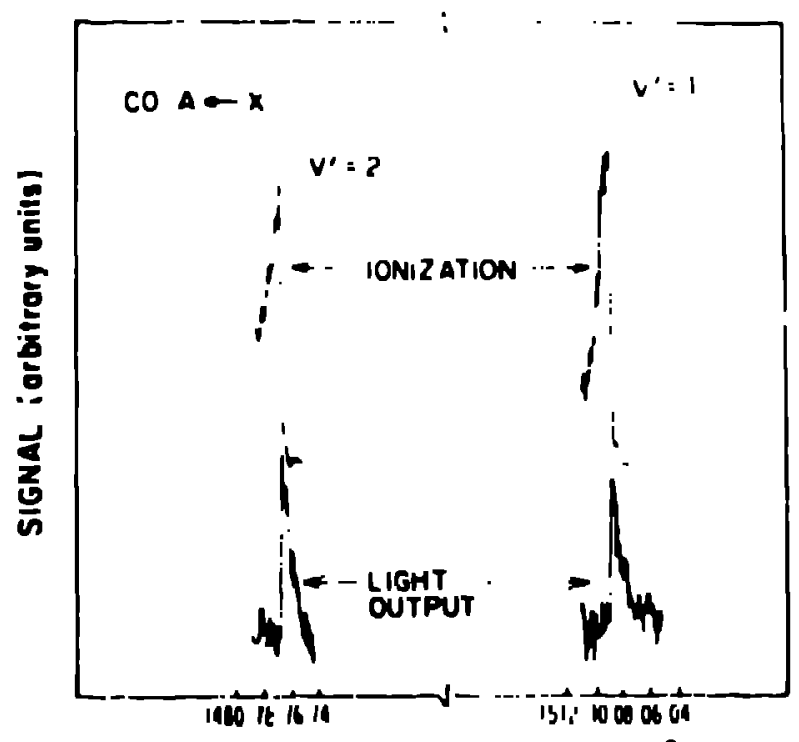

1/3 EXCITATION WAVELENGTH ( $\boldsymbol{A}$ )

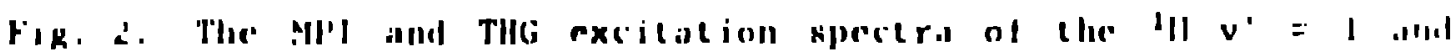
$v^{\prime}=2$ vilironic levels ill $(0)$ at a plessure of lo line. Al

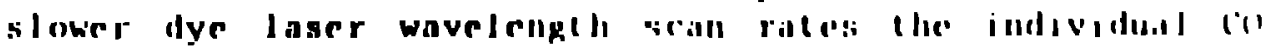

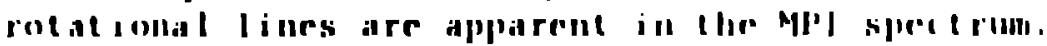

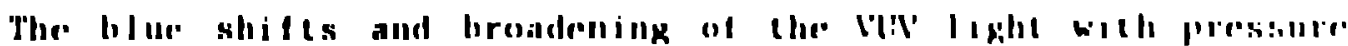

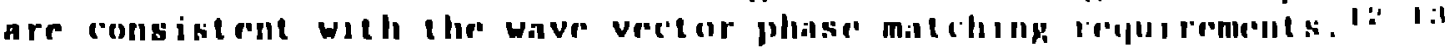
'The phisse mismatch $\Delta k$ mily he writcen as

$$
\Delta k=6 n\left({ }_{1},-n^{\prime}{ }^{\prime}\right) / \lambda_{p}
$$




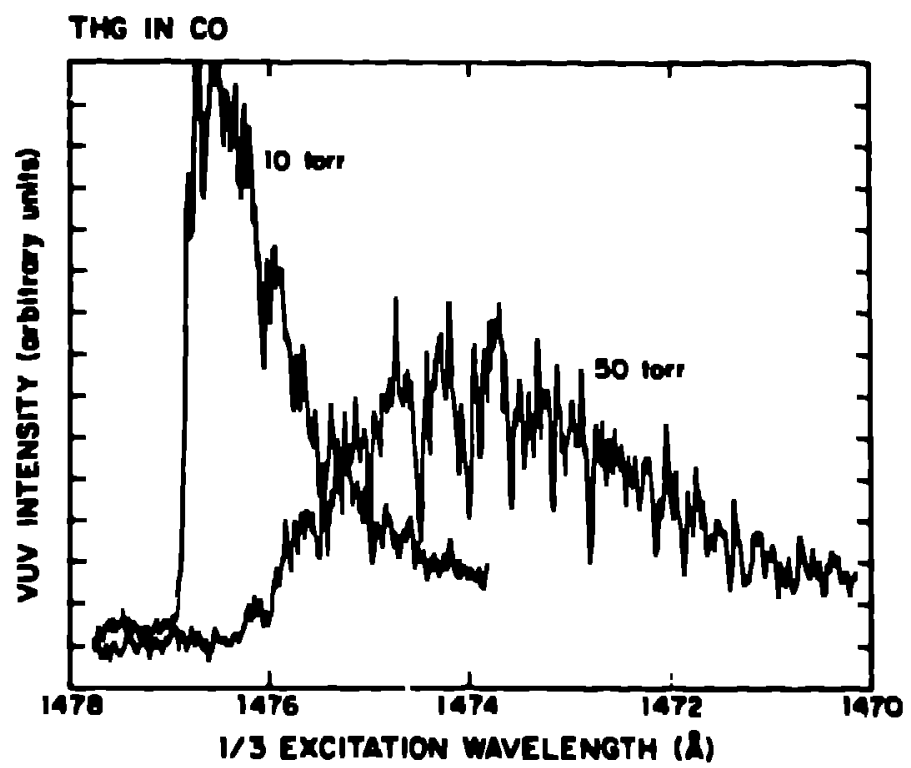

Fig. 3. Excitation spectra of the thirt-hurmonic emissicn in the ${ }^{1} \Pi v^{\prime}=2$ level of co at pressures $u 10$ and 50 Torr. The sharp dips in the l'ti emission are duc to self ahsorption liy rovilironic levels in the $\left(\mathrm{s}^{\prime}-\left(v^{\prime}=4\right)\right.$ band of ciarbon monoxide.

where $\lambda_{p}$ is the dye laser pump wavelenglh, and np and $n_{\text {Th }}$ are che refractive indices de $\lambda_{p}$ and $\lambda_{p} / 3$ respectively. Hjorktundis has shown that the optimum phase mismatch for focused beams has a small neqative value. In co the generated lilv frequency is higher than that of the vibronic levels of the $A$ statc, and, therefore, the anumalous dispersion makes a negative $\Delta k$ possible.

Measurements of the marimum T!IG output of $C(O)$ and de show that the'y are comparable. The efficiency for TllG in Xe has been previously reported. 14 It is important to note that the efficiency for THG goes as the square of the pump laser flux, thus increased THG efficiency should be possible by raising the laser flux to the limit of diclertric breakdown. We observed that this limit is noticeably higher in $C O$ than Xe. Also, the higher ${ }^{2} \Pi$ vibronic levels up to the predissociation limit exhibit third-harmonic emission, and tuning in the 1:300-1500 $R$ wavelength region is observed as shown in Fig. 4. Ongoing experiments are exploring the us: of co as a nonlinear medium to generate VW radiation in the Lymantalpha wavelength region. The $v^{\prime}=14$ level of ${ }^{13} \mathrm{CO}$ is ideally suited for this purpose. llsing an injection-locked XeC] laser as a source of $308 \mathrm{~nm}$ raliation, which is a near two-photon $A{ }^{\prime} I I \quad\left(v^{\prime}=0\right)$ resonillce, together with $578 \mathrm{~nm}$ Khodamice or copper vapor laser photons, a powerful 1216 \& source appears feasible. 
EXPEAMENTALLY OBSERVED THG OUTPUT

IN 5 - 350 TOAR ${ }^{12} \mathrm{CO}$ OR ${ }^{12} \mathrm{CO}$

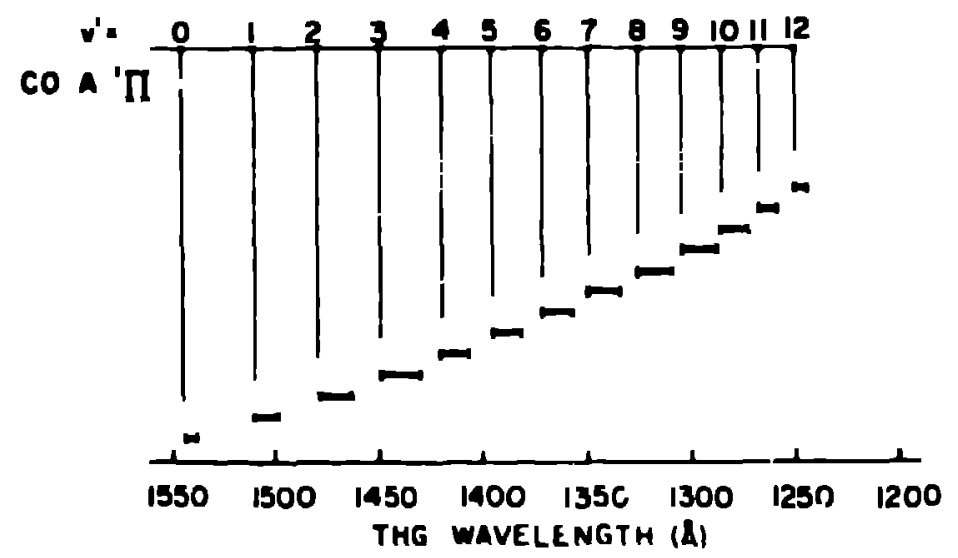

Fir. 4. havelenglhs for which third-harmonic iadialion has liech ohserved in $c^{12}\left(1^{16}\right.$ and for which Lhird-harmonis should be ohservad in $\mathrm{C}^{13()^{16}}$ using a single dỵe laser as an excilation source.

\section{SLMMARI}

In summary, it has been shown that $C(1$ vapor can be used to generate coherent tunable VU' raliation by THG using a single tullable dye laser. By simultaneously recording MPI and THG, it is found that these are competilive processes. The shifts to higher 1 requencies and the hroadening of the third-harmonic emission with increasing CO pressure dre found to be due to the phase matching requirements.

We are indebeed to J. R. Ackerhale for helpful discussions. Rescarch is sponsored by the Department of Energy under contract W-7405-eng-36 with the University of Cal.Inrnia.

\section{REFERFNCES}

1. S. C. Wallace, in Photoselectiuc Chcmistry Pt. 2, edited by .J. Jurener, R. D. Levine, and S. A. Rice (Wiley, New York, 1981), pp. $169-170$.

2. K. K. Innes, B. P. Stoichetf, and S. C. Wallacr, Appl. Phys. Lete. 29, $715(1976)$; S. C. Wallace anil K. K. Inues, J. Chem. Phys. $72,4805(1980)$.

3. J. C. Miller, R. N. Complon, M. G. Paync, and W. K. liarretl, Phys. Rev. LelL. 45, 114 (1980). 
4. M. G. Payne, W. R. Garrett, and H. C. Baker, Chen. Phys. Lett. 75, 468 (1980).

5. W. G. Rado, Appl. Phys. Lett. 11, 123 (1967).

6. P. H. Krupenie, The Band Spectrum of Carbon Monoxide, Nat. Bur. Stand. (US), NSRDS-5 (US GPO, Washington, D.C., 1966).

7. A. E. Douglas and C. K. Moller, Can. J. Phys. 33, 125 (1955).

B. J. D. Simons, A. M. Bass, and S. G. Tilford, Astrophys. J. 155, 345 (1969).

9. J. A. R. Samson and R. B. Cairns, GCA Technical Report No. 66-17-N (GCA Corp., Bedford, MA, 1966).

10. M. A. Duncan, T. G. Dietz, and R. E. Smalley, Chem. Phys. 44. 415 (1979).

11. P. C. Engleking, Chem. Phys. Lell. iz. 207 (1980).

12. G. C. Hjorklund, IEFE J. Uuantum Electroll. QE-11, 287 (1975).

13. D. C. Hanna, M. A. Puratich, and D. Colcer in Nonlinear Optics (.) Free Alons and Molecules, ediled hy D. L. MacAdam (SpringerTerlag. New York, 1979), Chap. is.

14. H. Hallig and R. hillenstejn, IEEE J. Quantum Electron. QE-17. $1566(1981)$. 